\title{
Fusobacterium nucleatum promotes chemoresistance to 5 -fluorouracil by upregulation of BIRC3 expression in colorectal cancer
}

Sheng Zhang ${ }^{1,2}$, Yongzhi Yang ${ }^{1,2}$, Wenhao Weng ${ }^{3,4}$, Bomin Guo ${ }^{5}$, Guoxiang Cai ${ }^{1,2}$, Yanlei Ma ${ }^{1,2^{*}}$ and Sanjun Cai ${ }^{1,2^{*}}$

\begin{abstract}
Background: Emerging evidence suggests a potential relationship between gut microbiota and the host response to chemotherapeutic drugs including 5 -fluorouracil (5-Fu). Fusobacterium nucleatum (Fn) has been linked to the initiation and progression of colorectal cancer (CRC). Unfortunately, little was known about the relationship between Fn infection and chemotherapeutic efficacy. Here, we investigate the potential relationship between $F n$ infection and chemotherapeutic efficacy of 5-Fu in CRC.

Methods: Differentially expressed genes of CRC cell lines induced by Fn infection were analyzed based on a whole genome microarray analysis Then, we explored the relationship between upregulation of BIRC3 induced by Fn infection and chemoresistance to 5-Fu in vitro and in vivo. Furthermore, we dissected the mechanisms involved in Fninduced BIRC3 expression. Finally, we investigated the clinical relevance of Fn infection, BIRC3 protein expression and chemoresistance to 5-Fu treatment in CRC patients.

Results: BIRC3 was the most upregulated gene induced by Fn infection via the TLR4/NF-KB pathway in CRC cells; Fn infection reduced the chemosensitivity of CRC cells to 5-Fu through upregulation of BIRC3 in vitro and in vivo. High Fn abundance correlated with chemoresistance in advanced CRC patients who received standard 5-Fu-based adjuvant chemotherapy after radical surgery.
\end{abstract}

Conclusions: Our evidence suggests that Fn and BIRC3 may serve as promising therapeutic targets for reducing chemoresistance to 5-Fu treatment in advanced CRC.

Keywords: Fusobacterium nucleatum, BIRC3, 5-fluorouracil, Chemoresistance, Colorectal cancer

\section{Background}

Colorectal cancer (CRC) is the third most common cancer and the second leading cause of cancer-related death, accounting for 1.4 million new cases and 0.6 million deaths worldwide in 2012 [1]. In China, there were more than 0.37 million new cases of CRC that led to more than 0.19 million deaths in 2015 [2]. Recently, accumulating evidence has shown that intestinal bacteria dysbiosis is related to the initiation and development of CRC [3-5]. Moreover, the gut microbiota can also influence

\footnotetext{
* Correspondence: yanleima@fudan.edu.cn; sanjuncai@hotmail.com ${ }^{1}$ Department of Colorectal Surgery, Fudan University Shanghai Cancer Center, 270 Dong'an Road, Shanghai 200032, China

Full list of author information is available at the end of the article
}

the efficacy of antitumor immunotherapy drugs [6-9] and have an impact on the treatment of CRC [10]. Interestingly, two studies have shown that bacterial metabolism can affect the host response to chemotherapeutic drugs including 5-fluorouracil (5-Fu) in C. elegans [11, 12]. Fusobacterium nucleatum (Fn), an anaerobic bacterium parasitic in the oral cavity, is increasingly linked to CRC [13-18]. However, few studies have focused on the impact of $F n$ on the treatment of CRC.

The inhibitor of apoptosis proteins (IAPs) are characterized by the presence of baculoviral IAP repeat (BIR) domains which are important for the binding and inhibition of caspases [19-21]. They can promote the survival of tumor cells and induce chemoresistance [22]. Therefore,

(C) The Author(s). 2019 Open Access This article is distributed under the terms of the Creative Commons Attribution 4.0 International License (http://creativecommons.org/licenses/by/4.0/), which permits unrestricted use, distribution, and 
IAPs have attracted wide attention as potential targets for cancer therapy [23]. BIRC3 is a member of the IAP family that can inhibit apoptosis by directly inhibiting the caspase cascade [24, 25]. BIRC3 can also contribute to chemoresistance in malignancies including CRC [26]. Our previous study using microarray analysis showed that $F n$ can significantly induce BIRC3 expression in CRC cell lines. Based on this finding, we hypothesize that the significant upregulation of BIRC3 expression induced by $F n$ might be responsible for chemoresistance in CRC.

In this study, we demonstrate that $F n$ infection reduced the chemosensitivity of CRC cells to 5 -Fu through upregulation of BIRC3 in vitro and in vivo, and high $F n$ abundance correlates with chemoresistance in advanced CRC patients who received standard 5-Fu-based adjuvant chemotherapy after radical surgery. Our evidence suggests that $F n$ and BIRC3 may serve as promising therapeutic targets for reducing chemoresistance to 5 -Fu treatment in advanced CRC.

\section{Methods}

\section{Bacteria strains and cell lines}

Fn strain ATCC 25586 was purchased from American Type Culture Collection (ATCC) and grown in Columbia blood agar (Sigma, USA) in an anaerobic bag (Merier, France) at $37^{\circ} \mathrm{C}$ as previously described [15]. HCT116, HT29 and $293 \mathrm{~T}$ cells were obtained from GeneChem and cultured in DMEM-F12(Gibco, USA) supplemented with $10 \%$ FBS (Gibco) and $1 \%$ penicillin and streptomycin (Beyotime, China) at $37^{\circ} \mathrm{C}$ in a humidified $5 \% \mathrm{CO} 2$ atmosphere. For $F n$ infection assay, cells were cultured in medium without antibiotics and incubated with $F n$ at a multiplicity of infection (MOI) of 100:1 as previously described [27].

\section{Patients and specimens}

A total of 94 patients diagnosed with advanced CRC were included in this study. All the patients received standard 5-Fu-based adjuvant chemotherapy after radical surgery in Fudan University Cancer Center from 2007 to 2017. None of them received preoperative treatment. Ninety-four formalin-fixed paraffin-embedded (FFPE) CRC tissues were obtained from the pathological archives. Prognostic information was collected by the medical record system and telephone follow-up. The median follow-up time was 38.5 months, ranging from 7 to 132 months. During the follow-up period, 45 patients (47.8\%) suffered from recurrence of the disease. Clinicopathological data of the patients are summarized in Table 1. Written informed consent was obtained from the patients, and the study was approved by the Ethics Committee of the hospital.

\section{Quantitative real-time PCR}

For detection of $F n$ abundance, genomic DNA (gDNA) was extracted from FFPE tissues with QIAamp DNA FFPE Tissue Kit (QIAGEN, Germany). The abundance of $F n$ was determined by detecting the $16 \mathrm{~S}$ gene using qPCR. Each $10-\mu \mathrm{L}$ reaction contained 80 ng of gDNA, $0.4 \mathrm{mM}$ each primer and $1 \times$ final concentration of SYBR Green PCR Master Mix (Thermo Fisher Scientific, USA). Amplification was performed using the ABI Step One Plus Real-Time PCR System (Applied Biosystems, USA) under the following reaction conditions: $10 \mathrm{~min}$ at $95^{\circ} \mathrm{C}$, followed by 40 cycles of $95^{\circ} \mathrm{C}$ for $15 \mathrm{~s}$ and at $60^{\circ} \mathrm{C}$ for $1 \mathrm{~min}$. The gene prostaglandin transporter (PGT) was used as the internal reference as previously described [13]. For detection of target gene expression, total RNA was isolated using TRIzol reagent (Invitrogen, USA), and $1 \mathrm{mg}$ of total RNA was reverse transcribed using a reverse transcription kit (Promega, USA). Complementary DNA (cDNA) was amplified and quantified on the ABI Step One Plus Real-Time PCR System (Applied Biosystems) using SYBR Green (Takara, China) under the following reaction conditions: $30 \mathrm{~s}$ at $95^{\circ} \mathrm{C}$ and 40 cycles of $5 \mathrm{~s}$ at $95^{\circ}$ $\mathrm{C}$ and $30 \mathrm{~s}$ at $60^{\circ} \mathrm{C}$. Q-PCR was performed in triplicate for each sample, and the $2^{-\Delta \Delta C t}$ method was used to assess the relative expression levels of target genes as previously described [28]. GAPDH served as an internal reference gene and all the primer sequences are given in Additional file 1: Table S1.

\section{Microarray analysis}

Total RNA was isolated from HCT116 cells infected with or without $F n$ for $24 \mathrm{~h}$. Then, the probes were prepared by labeling the mRNA with Cy5-dUTP (for the control group) or with Cy3-dUTP (for the infected group) through reverse transcription. The labeled cDNA probes were hybridized to a Human Whole Genome Microarray (Zhuolibiotech, China) and scanned with an Axon 4000 scanner (Molecular Devices, Sunnyvale, CA, USA). Bioinformatics analysis was performed. The list of differentially expressed genes was filtered for fold changes $>2.0$, and those genes were selected for further investigation.

\section{Cell proliferation assay and drug cytotoxicity assay}

The percentage of viable cells was determined by Cell Counting Kit-8 (CCK8; Yeasen, China) assay under different treatment conditions. Briefly, cells were seeded in 96-well plates with $100 \mu \mathrm{L}$ of culture medium. Ten microliters of CCK8 solution was added to each well at the indicated time points and incubated at $37^{\circ} \mathrm{C}$ for $2 \mathrm{~h}$. The reaction product was assessed by measuring the optical absorbance at $450 \mathrm{~nm}$ using a spectrophotometer (Thermo Fisher Scientific, USA). 
Table 1 Clinicopathological characteristics of CRCs in according to Fn abundance or recurrence status

\begin{tabular}{|c|c|c|c|c|c|c|c|}
\hline Characteristics & $\begin{array}{l}\text { All cases } \\
(n=94)\end{array}$ & $\begin{array}{l}\text { Fn-low/negative } \\
(n=73)\end{array}$ & $\begin{array}{l}\text { Fn-high } \\
(n=21)\end{array}$ & $P$ value & $\begin{array}{l}\text { Non-recurrence } \\
(n=49)\end{array}$ & $\begin{array}{l}\text { Recurrence } \\
(n=45)\end{array}$ & $P$ value \\
\hline Age & & & & 0.849 & & & 0.646 \\
\hline$\geq 60$ & $42(44.7 \%)$ & $33(45.2 \%)$ & 9 (42.9\%) & & $23(46.9 \%)$ & 19 (42.2\%) & \\
\hline$<60$ & $52(55.3 \%)$ & 40 (54.8\%) & $12(57.1 \%)$ & & 26 (53.1\%) & $26(57.8 \%)$ & \\
\hline Sex & & & & 0.936 & & & 0.323 \\
\hline Female & 41 (43.6\%) & $32(43.8 \%)$ & 9 (42.9\%) & & 19 (38.8\%) & 22 (48.9\%) & \\
\hline Male & 53 (56.4\%) & $41(56.2 \%)$ & $12(57.1 \%)$ & & 30 (61.2\%) & $23(51.1 \%)$ & \\
\hline Tumor location & & & & 0.051 & & & 0.079 \\
\hline Rectum & $54(57.4 \%)$ & $42(57.5 \%)$ & $12(57.1 \%)$ & & $23(46.9 \%)$ & 31 (68.9\%) & \\
\hline Distal colon & $23(24.5 \%)$ & $21(28.8 \%)$ & $2(9.5 \%)$ & & $16(32.7 \%)$ & 7 (15.6\%) & \\
\hline Proximal colon & 17 (18.1\%) & 10 (13.7\%) & 7 (33.3\%) & & 10 (20.4\%) & $7(15.6 \%)$ & \\
\hline Tumor size & & & & 0.156 & & & 0.131 \\
\hline$>4 \mathrm{~cm}$ & $41(43.6 \%)$ & 29 (39.7\%) & $12(57.1 \%)$ & & 25 (51.0\%) & 16 (35.6\%) & \\
\hline$\leq 4 \mathrm{~cm}$ & $53(56.4 \%)$ & 44 (60.3\%) & 9 (42.9\%) & & $24(49.0 \%)$ & 29 (64.4\%) & \\
\hline Differentiation & & & & 0.840 & & & 0.573 \\
\hline Well/Moderate & 75 (79.8\%) & 59 (80.8\%) & $16(76.2 \%)$ & & 38 (77.6\%) & 37 (82.2\%) & \\
\hline Poor & 19 (20.2\%) & 14 (19.2\%) & $5(23.8 \%)$ & & 11 (22.4\%) & $8(17.8 \%)$ & \\
\hline Vascular invasion & & & & 0.874 & & & 0.003 \\
\hline Positive & 30 (31.9\%) & 23 (31.5\%) & 7 (33.3\%) & & 9 (18.4\%) & $21(46.7 \%)$ & \\
\hline Negative & $64(68.1 \%)$ & 50 (68.5\%) & $14(66.7 \%)$ & & 40 (81.6\%) & $24(53.3 \%)$ & \\
\hline Neural invasion & & & & 0.808 & & & 0.029 \\
\hline Positive & $22(23.4 \%)$ & $18(24.7 \%)$ & $4(19.0 \%)$ & & 7 (14.3\%) & 15(33.3\%) & \\
\hline Negative & $72(76.6 \%)$ & 55 (75.3\%) & 17 (81.0\%) & & 42 (85.7\%) & $30(6.7 \%)$ & \\
\hline TNM stage & & & & 0.130 & & & 0.153 \\
\hline$\|$ & 45 (47.9\%) & $38(52.1 \%)$ & 7 (33.3\%) & & $20(40.8 \%)$ & 25 (33.3\%) & \\
\hline III & 49 (52.1\%) & 35 (47.9\%) & $14(66.7 \%)$ & & $29(59.2 \%)$ & $20(66.7 \%)$ & \\
\hline BIRC3 staining & & & & & & & 0.008 \\
\hline Positive & $36(38.3 \%)$ & $21(28.8 \%)$ & $15(71.4 \%)$ & 0.000 & 25 (51.0\%) & 11 (71.4\%) & \\
\hline Negative & $58(61.7 \%)$ & $52(71.2 \%)$ & $6(28.6 \%)$ & & $24(49.0 \%)$ & $34(28.6 \%)$ & \\
\hline TLR4 staining & & & & & & & 0.036 \\
\hline Positive & $50(53.2 \%)$ & 35 (47.9\%) & 15 (71.4\%) & 0.057 & $21(42.9 \%)$ & 29 (64.4\%) & \\
\hline Negative & 44 (46.8\%) & $38(52.1 \%)$ & $6(28.6 \%)$ & & $28(57.1 \%)$ & 16 (35.6\%) & \\
\hline Fn abundance & & & & & & & 0.014 \\
\hline Fn-low/negative & 73 (77.7\%) & & & & 43(83.6\%) & 30 (71.1\%) & \\
\hline Fn-high & $21(22.2 \%)$ & & & & $6(16.4 \%)$ & 15 (28.9\%) & \\
\hline
\end{tabular}

Pearson Chi-square test

\section{Western blot analysis}

Total cellular protein was extracted and separated by $10 \%$ SDS-PAGE then transferred to polyvinylidene fluoride (PVDF) membranes. Membranes were blocked with $5 \%$ BSA in TBST for $1 \mathrm{~h}$ at room temperature and then incubated with the primary antibodies overnight at $4{ }^{\circ} \mathrm{C}$, followed by incubation with secondary antibodies for $2 \mathrm{~h}$ at room temperature. The signals were detected using an enhanced chemiluminescence reagent (Millipore,
USA). The information for all antibodies used is listed in Additional file 2: Table S2.

\section{Immunohistochemical staining and immunofluorescence} staining

For immunohistochemistry (IHC), an EnVision+ Dual Link Kit (Dako, USA) was used according to the manufacturer's instructions. The staining process was performed as previously described [29]. All sample slides 
were scored separately by two pathologists according to the distribution, intensity and percentage of positive cells [30]. Briefly, the staining intensity was scored as 0 (negative), 1 (weak), 2 (medium) or 3 (strong). The extent of staining was scored as $0(<5 \%), 1(5-25 \%), 2(26-50 \%)$, $3(51-75 \%)$ or $4(>75 \%)$. The scores of intensity and extent were multiplied to generate the immunoreactivity score. Final staining scores $0-4$ and 5-12 were considered negative and positive, respectively.

$F n$-induced NF- $\mathrm{KB}$ activation was detected using an NF-kB Activation Nuclear Translocation Assay Kit (Beyotime, China) according to the manufacturer's instructions [31]. Briefly, cells were fixed and washed followed by incubation with blocking buffer for $1 \mathrm{~h}$. Then, cells were incubated with the primary antibody against NF-kB P65 overnight in a humidified chamber at $4{ }^{\circ} \mathrm{C}$ before a Cy3-conjugated secondary antibody was applied for $1 \mathrm{~h}$ at room temperature. Finally, nuclei of cells were stained with DAPI for $5 \mathrm{~min}$. Images were captured by laser confocal microscopy at an excitation wavelength of $350 \mathrm{~nm}$ for DAPI (blue) and $540 \mathrm{~nm}$ for Cy3 (red), and merged using software.

\section{Apoptosis detection}

The cell apoptosis ratio was determined by flow cytometric analysis with an Annexin V FITC/PI double stain assay (BD Biosciences, USA) according to the manufacturer's instructions. Levels of apoptosis in the xenograft tumor tissues were determined by terminal deoxynucleotidyl transferasemediated dUTP nick end labeling (TUNEL) technology using an Apoptosis Assay Kit (Beyotime, China).

\section{Small interfering RNA (siRNA) silencing in vitro}

SiRNAs were purchased from GenePharma (Shanghai, China). Transfection of siRNA was performed using Lipofectamine 3000 (Invitrogen, USA) according to the manufacturer's protocol and nonspecific siRNAs were used as negative controls. The sequences of siRNA used in this study are shown in Additional file 1: Table S1.

\section{Chromatin immunoprecipitation assay (ChIP)}

ChIP assays were carried out using a ChIP Assay Kit (Beyotime, China) following by the manufacturer's protocol. Briefly, cells were treated with $1 \%$ formaldehyde to cross-link DNA and proteins. Afterwards, the cell lysates were sonicated to generate chromatin fragments of 200$300 \mathrm{bp}$ and immunoprecipitated with an anti-P65 antibody or IgG as a control. The region $5 \mathrm{~kb}$ upstream of BIRC3 gene was set as a negative control. The precipitated chromatin DNA was recovered and measured by qPCR. The percent input for each experiment was converted to fold change relative to an untreated control. Average fold changes from 3 independent experiments were then plotted. Primer sequences for the ChIP-qPCR assay are listed in Additional file 1: Table S1.

\section{Dual luciferase reporter assay}

The NF-кB P65 binding motif in the promoter region of BIRC3 was predicted by JASPAR (http://jaspar.genereg.net/). Two putative binding sites with the highest score were selected. Wild-type (WT) or mutated putative NF-kB P65 binding sites in the BIRC3 promoter region were amplified and cloned into the pGL3-Promoter vector (Additional file 3: Figure S1). 293 T cells were transfected with WT or mutated BIRC3 promoter construct and pcDNA-P65 or pcDNA-NC vector (GeneChem, China) using Lipofectamine 3000 (Invitrogen, USA). Luciferase activity was determined using a Dual Luciferase Reporter Assay Kit (Promega, USA) according to the manufacturer's protocol.

\section{Mouse model}

For the xenograft experiments, 5-week-old male BALB/ c nude mice (Chinese Academy of Sciences, Shanghai, China) were housed in laminar flow cabinets under specific pathogen-free conditions. To establish the CRC xenograft model., HCT116 $\left(5 \times 10^{6}\right.$ cells $\left./ 0.5 \mathrm{ml}\right)$ cells were subcutaneously injected into the right flank of the mice. One week after subcutaneous inoculation, mice were randomly divided into 4 groups for different sets of experiments $(n=5)$. Fn and siRNA were administered by multipoint intratumor injection every 3 days for a total of five injections using an in vivo transfection reagent (Entranster ${ }^{\text {rm }}$-in vivo, Engreen, China) according to the instructions [32]. 5-Fu (Absin, China) was administered by an intraperitoneal injection of $5 \mathrm{mg} / \mathrm{kg}$ per mouse, twice per week for 2 weeks. Tumor volume (V) was calculated using the following formula: $\mathrm{V}=\left(\mathrm{a} \times \mathrm{b}^{2}\right) / 2$, where $\mathrm{a}$ and $\mathrm{b}$ represent the longer and shorter tumor diameters, respectively. After the 2-week treatment, all mice were sacrificed and the tumors were collected. The samples were fixed with formalin for IHC, TUNEL assay and hematoxylin and eosin (H\&E) staining. The animal study was approved by the Committee on Animals Handling of Fudan University Shanghai Cancer Center.

\section{Statistical analysis}

Statistical analyses were performed using GraphPad Prism software 7.0 (GraphPad Software, La Jolla, CA). Data are presented as the means \pm standard deviation. Unpaired Student $\mathrm{t}$ test or nonparametric Mann-Whitney test was applied to assess the statistical significance between groups with different treatment. Correlations were calculated according to Pearson Chi-square test All statistical tests were two-sided and $p<0.05$ was considered statistically significant. 


\section{Results}

Fn infection significantly induces expression of BIRC3 in CRC cell lines

To assess the global effect of $F n$ infection in vitro, human CRC HCT116 cells were incubated with or without $F n$ for $24 \mathrm{~h}$. Whole genome microarray analysis was performed and differentially expressed genes were analyzed. The result showed that BIRC3 was the gene with the highest expression (12-fold increase) induced by $F n$ infection compared with the control groups (Fig. 1a). To validate the upregulation of BIRC3 in CRC cell lines infected with $F n$, qPCR and Western blot analysis were performed. The results showed that the mRNA levels of BIRC3 in HCT116 and HT29 cells were significantly upregulated by $F n$ infection for $24 \mathrm{~h}$ and $48 \mathrm{~h}$ respectively (Fig. 1b). Western blot analysis also showed that Fn infection increased the expression of BIRC3protein at different time intervals (Fig. 1c). As BIRC3 is a member of the IAP family [33], we also examined the protein levels of the other members of this family including BIRC2 (cIAP1), BIRC4 (XIAP) and BIRC5 (Survivin). The results showed that the protein levels of these genes was not affected by $F n$ infection (data not shown).

\section{Fn induces chemoresistance of CRC cells to 5-Fu via upregulation of BIRC3 in vitro}

Since BIRC3 is a member of the IAP family that inhibits apoptosis by directly inhibiting caspase cascade [25], and can contribute to chemoresistance in malignancies including CRC [26, 34], we speculated that the upregulation of BIRC3 expression induced by $F n$ infection may lead to chemoresistance in CRC. To test this hypothesis, we initially determined whether $F n$ infection decreased cytotoxicity induced by 5 -Fu in CRC cells. The results showed that Fn decreased cytotoxicity induced by 5-Fu in HCT116 and HT29 cells (Fig. 2a). We also found that the $50 \%$ inhibitory concentration $\left(\mathrm{IC}_{50}\right)$ of $5-\mathrm{Fu}$ in HCT116 and HT29 cells was approximately $30 \mu \mathrm{M}$ and $20 \mu \mathrm{M}$, respectively (Fig. 2a).

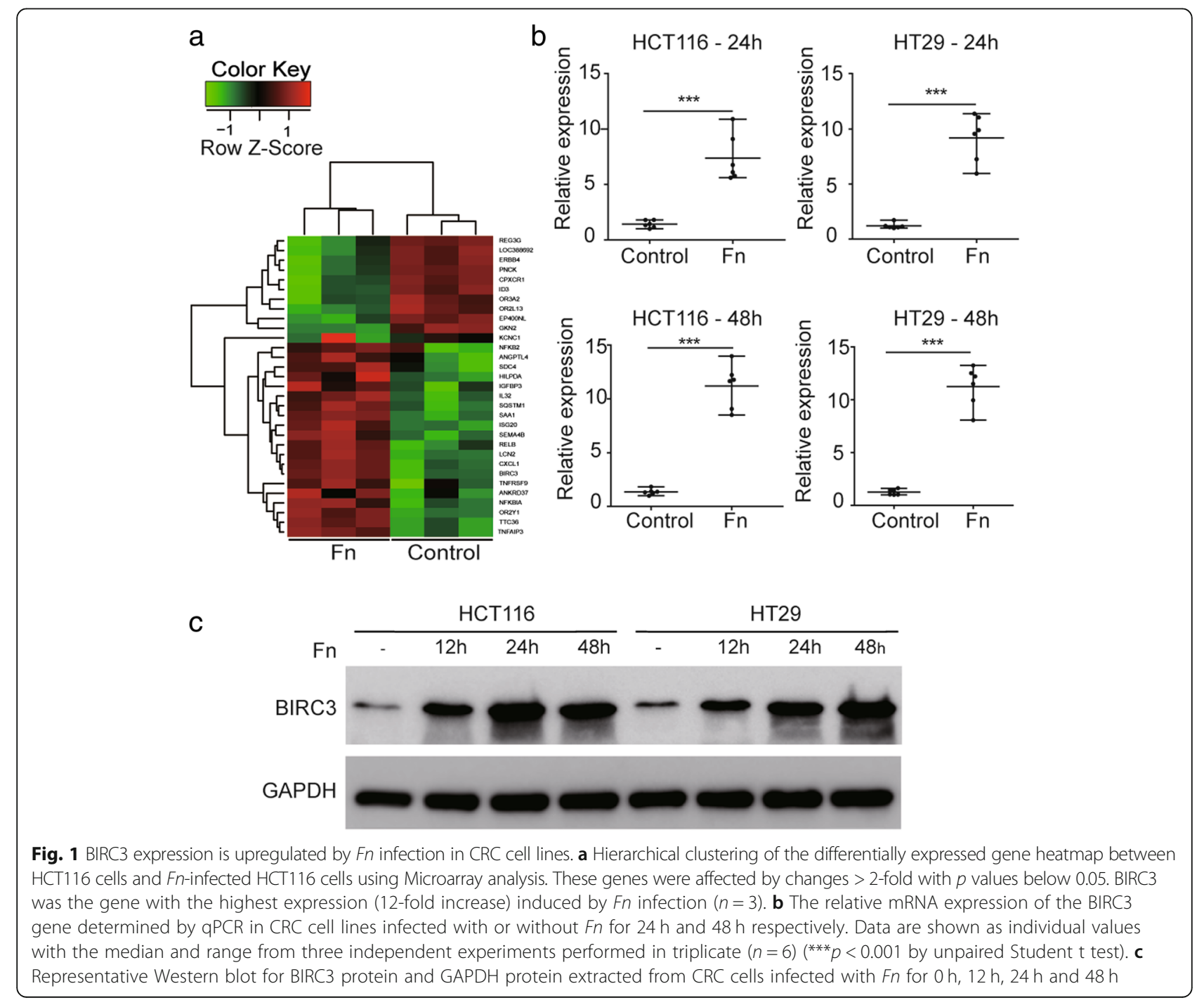




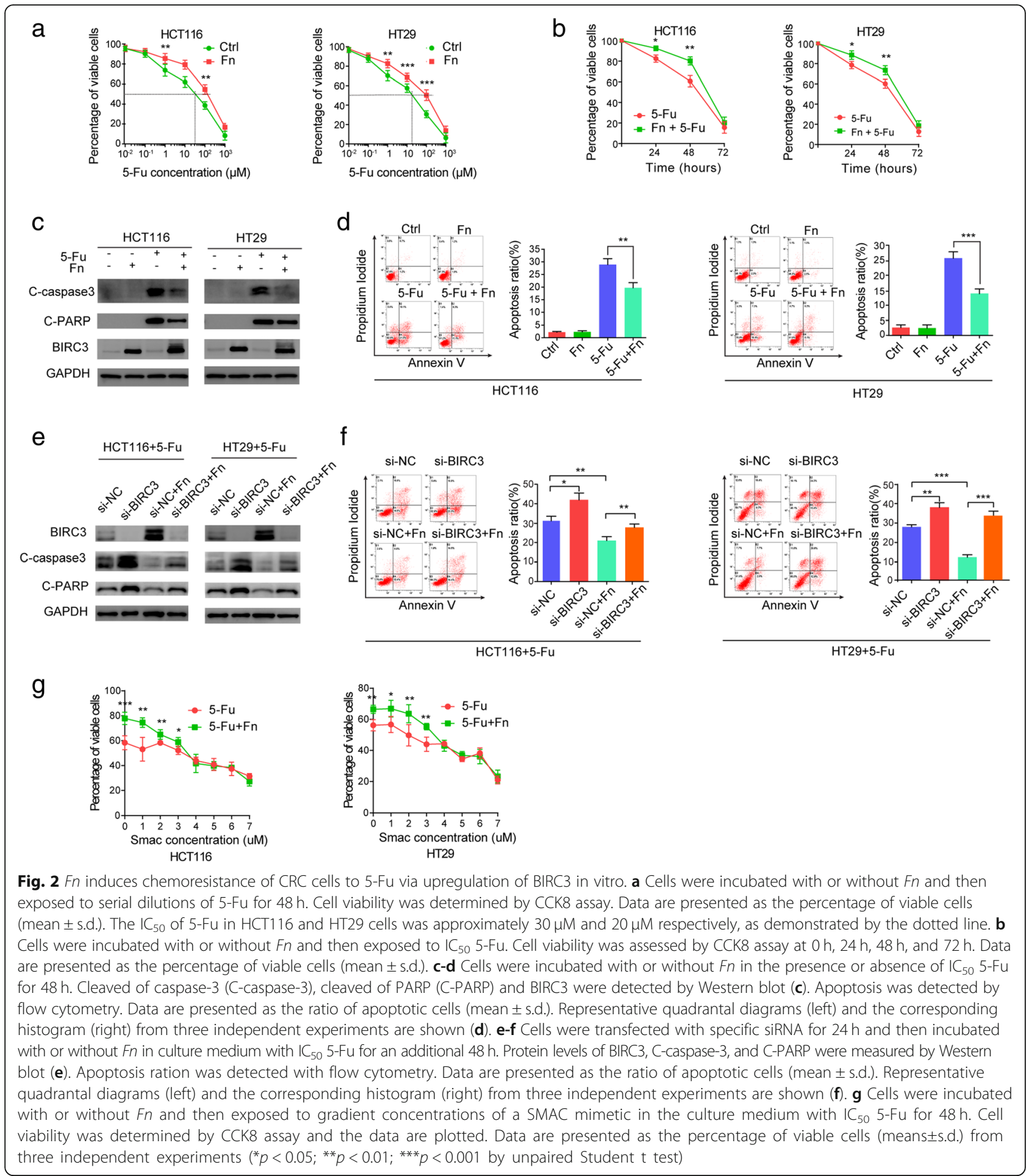

Based on this result, cells were incubated with or without $F n$ and then exposed to $\mathrm{IC}_{50} 5$ - $\mathrm{Fu}$ and cell viability was assessed at the indicated time. Consistent with previous results, $F n$ infection significantly reduced cytotoxicity induced by 5 -Fu treatment for $24 \mathrm{~h}$ or $48 \mathrm{~h}$ (Fig. $2 \mathrm{~b}$ ). To address whether $\mathrm{Fn}$ infection protects $\mathrm{CRC}$ cells from 5-Fu- mediated apoptosis, we cocultured HCT116 and HT29 cells with or without $F n$ in the presence or absence of $\mathrm{IC}_{50}$ 5-Fu for $48 \mathrm{~h}$. Western blotting showed that the cleavage of caspase3 (C-caspase3) and cleavage of PARP (C-PARP) was induced by $5-\mathrm{Fu}$, while this effect was attenuated by $\mathrm{Fn}$ infection (Fig. 2c). Flow cytometry analysis with Annexin V 
FITC/PI double stain assay was performed to assess the apoptosis ratio of each group. The results showed that 5-Fu-induced apoptosis was crippled by $F n$ infection (Fig. 2d). Taken together, these results suggest that $F n$ infection can reduce the chemosensitivity of CRC cells to 5 - Fu in vitro.

To confirm the role of BIRC3 in the Fn-induced chemoresistance to 5-Fu, BIRC3 gene was silenced in CRC cells using specific siRNA. Cells were incubated with or without $F n$ and then exposed to $\mathrm{IC}_{50} 5-\mathrm{Fu}$ for $48 \mathrm{~h}$. Western blotting showed that $F n$ infection crippled the level of C-caspase3 and C-PARP induced by 5 -Fu, while this effect was abolished by silencing the BIRC3 gene in the cells (Fig. 2e). Under the same conditions, the apoptosis ratio of each group was detected with flow cytometry. We also observed that silencing of BIRC3 abrogated $F n$-induced chemoresistance of the cells in response to 5-Fu (Fig. 2f). Furthermore, a small molecule antagonist of BIRC3 named SMAC mimetic (SM-406, Selleck, China) was used to antagonize the effect of BIRC3 [35, 36]. The results showed that the chemoresistance effect mediated by $F n$ gradually weakened with increasing of SMAC mimetic concentration in the cells (Fig. 2g). Altogether, these data strongly suggest that BIRC3 plays an essential role in the $\mathrm{Fn}$-induced chemoresistance of $\mathrm{CRC}$ cells in response to 5 - $\mathrm{Fu}$ in vitro.

\section{The TLR4/NF-KB pathway regulates BIRC3 expression in CRC cells cocultured with Fn}

It was previously reported that $F n$ infection can activate the TLR4/NF- $\mathrm{KB}$ pathway in CRC cells $[17,37]$ and bioinformatics analysis of the microarray data also supported this conclusion. In addition, NF- $\mathrm{KB}$ activation induces the expression of a variety of target genes including BIRC3 [38, 39]. Based on this evidence, we speculated that the TLR4/NF-kB pathway may be involved in the $F$-induced upregulation of BIRC3. First, we observed NF- $\kappa$ B P65 nuclear translocation when the cells were incubated with $F n$ for $2 \mathrm{~h}$. (Fig. 3a). Western blot analysis showed that $F n$ infection contributed to NF- $k B$ P65 activation and upregulation of BIRC3, while silencing of NF-kB P65 abrogated the $F n$-mediated upregulation of BIRC3 in the cells. (Fig. 3b). To examine the direct interaction between NF-kB P65 and the BIRC3 promoter region, a dual luciferase reporter assay was performed. The result showed that cotransfection of plasmids containing transcription factor P65 (TF-P65) and the wild-type (WT) pGL3-Promoter vector resulted in a significant increase in promoter activity compared to the transcription factor-negative (TF-NC) group (Fig. $3 \mathrm{~d}$ ), suggesting that NF- $\mathrm{kB}$ P65 positively regulates the activity of the BIRC3 promoter. To further explore the potential NF- $\mathrm{kB}$ P65 binding sites on the BIRC3 promoter region, two mutant type (MT) pGL3-Promoter vectors were generated (Fig. 3c and Additional file 3: Figure S1) and independently cotransfected with TF-P65 or TF-NC. We observed that cotransfection of the pGL3-MT2-Promoter plasmid resulted in the same effect on promoter activity as that with the pGL3-WTPromoter plasmid, while this effect was not observed with the cotransfection of the pGL3-MT1-Promoter plasmid (Fig. 3d). Therefore, we may infer that the binding site is located 156 to $165 \mathrm{bp}$ upstream of the transcription start site (TSS) (Fig. 3c). Furthermore, ChIP assays were performed for P65 in CRC cells. We found that recruitment of $\mathrm{P} 65$ to the BIRC3 promoter region was enhanced by $F n$ infection in the cells (Fig. 3e). To investigate whether TLR4 and MyD88 are involved in the Fn-mediated upregulation of BIRC3, we first confirmed that $F n$ infection can upregulate the levels of TLR4 and MyD88 transcripts in the cells. (Fig. 3f). Next, cells were transfected with specific siRNA (si-TLR4 or si-NC) for $24 \mathrm{~h}$ followed by coculture with $F n$ for additional $48 \mathrm{~h}$. Western blotting analysis showed that silencing of TLR4 crippled Fn-mediated upregulation of BIRC3 in the cells.(Fig. 3g). Collectively, these results support the hypothesis that the $F n$ induces BIRC3 expression through TLR4/NF- $\mathrm{kB}$ pathway in CRC cells.

\section{Fn induces chemoresistance of CRC cells in response to 5- Fu in vivo}

A subcutaneous xenograft model was used to further explore whether $F n$ induces chemoresistance in CRC cells and to determine the role of BIRC3 in this effect in vivo. Four groups were designed according to the purpose of this experiment. The results showed that tumor growth was significantly decreased by 5 -Fu treatment while this effect was crippled by $F n$ infection in vivo (Fig. 4a-b). Interestingly, silencing of BIRC3 partially attenuated the $\mathrm{Fn}$-mediated chemoresistance of tumors in response to 5-Fu in vivo (Fig. 4a-b). These findings were further confirmed by TUNEL assay (Fig. 4c-d). To explore the relationship between $F n$ infection and the expression level of TLR4 and BIRC3 in vivo, IHC was carried out in tumor sections. We observed that $F n$ infection significantly upregulated the expression levels of TLR4 and BIRC3 (Fig. 4c-d). In conclusion, these data indicate that BIRC3 plays an essential role in the $F n$-mediated chemoresistance of CRC cells in response to 5 -Fu in vivo.

\section{High Fn abundance correlates with poor recurrence-free survival (RFS) in advanced CRC}

To investigate whether $F n$ infection and its downstream target genes can affect the efficacy of 5-Fu-based chemotherapy in CRC patients, we initially detected the Fn abundance and BIRC3 and TLR4 expression levels in FFPE CRC tissues from patients who were pathologically diagnosed with advanced CRC and received standard 

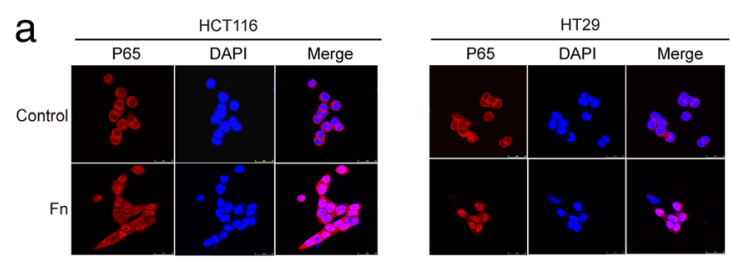

b

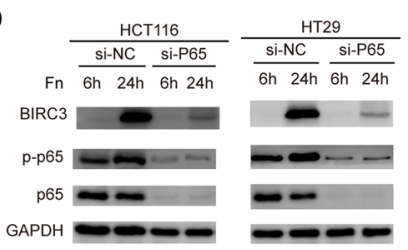

C

Prdicted NF-kB P65 binding sites

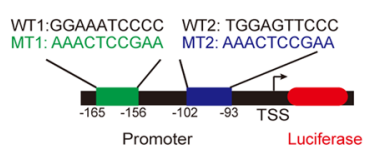

e

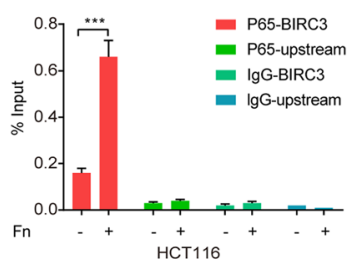

f
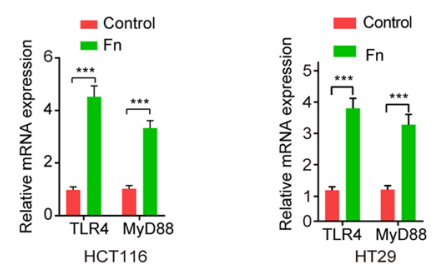

d
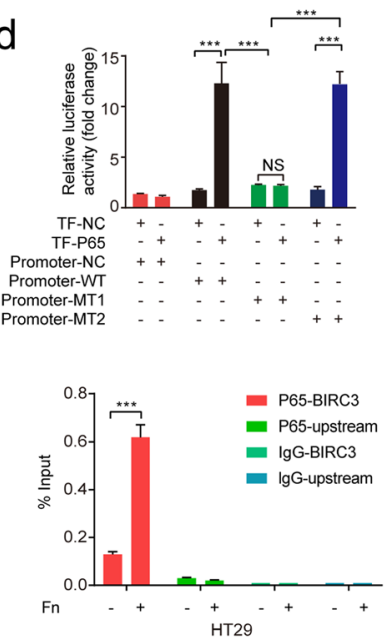

g

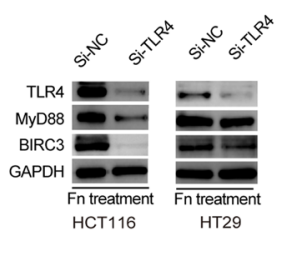

Fig. $3 \mathrm{Fn}$ induces BIRC3 expression through TLR4/NF-kb pathway. a Cells were cocultured with or without Fn for $2 \mathrm{~h}$ followed by incubation with an antibody against NF-KB P65 and a Cy3 fluorescein-conjugated secondary antibody, and nuclei were stained with DAPI. b Cells were transfected with specific siRNA (si-P65 or si-NC) for $24 \mathrm{~h}$ followed by cocultured with Fn for an additional $6 \mathrm{~h}$ or $24 \mathrm{~h}$. Protein levels of BIRC3, p-P65, and P65 were measured by Western blot. c Schematic representation of the location of two predicted NF-kB P65 binding motifs in the BIRC3 promoter region. WT: wild-type; MT1: mutant type 1; MT2: mutant type 2. d pGL3-Promoter reporter plasmids (NC, WT, MT1, MT2) and pcDNA3.1-TF-CDNA vectors (TF-NC, TF-P65) were generated. Dual luciferase reporter assay were carried out according to different combinations. TF: transcription factor; NS: nonsignificant. e Cells were cocultured with or without $F n$ for $2 \mathrm{~h}$. Then, ChIP assays were carried out with an anti-P65 antibody or rabbit IgG. qPCR was then carried out for the BIRC3 promoter region or the region $5 \mathrm{~kb}$ upstream of BIRC3 gene. $\mathbf{f} C$ ells were co-cultured with or without $F n$ for $24 \mathrm{~h}$. The relative mRNA level of TLR4 and MyD88 was detected by qPCR. $\mathbf{g}$ Cells were transfected with specific siRNA (si-TLR4 or si-NC) for $24 \mathrm{~h}$ followed by co-cultured with Fn for an additional $48 \mathrm{~h}$. Protein levels of TLR4, MyD88, and BIRC3 were measured by Western blot. (*** $p<0.001$ by unpaired Student's t-test)

5-Fu-based adjuvant chemotherapy after radical surgery. The distribution of $F n$ abundance is shown in Additional file 4: Figure S2. We set a cut-off value of $0.1\left(2^{-\Delta \mathrm{Ct}}\right)$ for $F n$ abundance and divided the patients into the Fn-low/ negative group and the $F n$-high group as previously described [40]. Based on this division, we identified 22.3\% of patients as having a high amount of $F n$. The expression level of TLR4 and BIRC3 in FFEP tissues were determined by IHC assay. and divided into positive or negative status. Representative IHC images of BIRC3 and TLR4 proteins in FFEP CRC tissues were shown in Fig. 5a (BIRC3) and Fig. 5b (TLR4). As shown in Table 1, BIRC3 expression level was positively correlated with $F n$ abundance $(71.4 \%$ in the $F n$-high group vs. $28.8 \%$ in the $F n$-low/negative group; $p<0.001)$. We also observed a trend of higher TLR4 expression in the Fn-high group (71.4\%) than in the Fn-low/negative group (47.9\%), although there was no statistical significance $(p=0.057)$. In line with our previous in vitro findings, these results suggested that $F n$ infection and its downstream target genes TLR4 and BIRC3 are clinically relevant in CRC patients.

Moreover, high $F n$ abundance $(p=0.014)$, high levels of TLR4 $(p=0.036)$ and BIRC3 $(p=0.008)$ proteins were more likely detected in patients with recurrence, compared with patients without recurrence.(Table 1). In addition, vascular invasion $(p=0.003)$ and neural invasion $(p=0.029)$ positively correlated with recurrence 


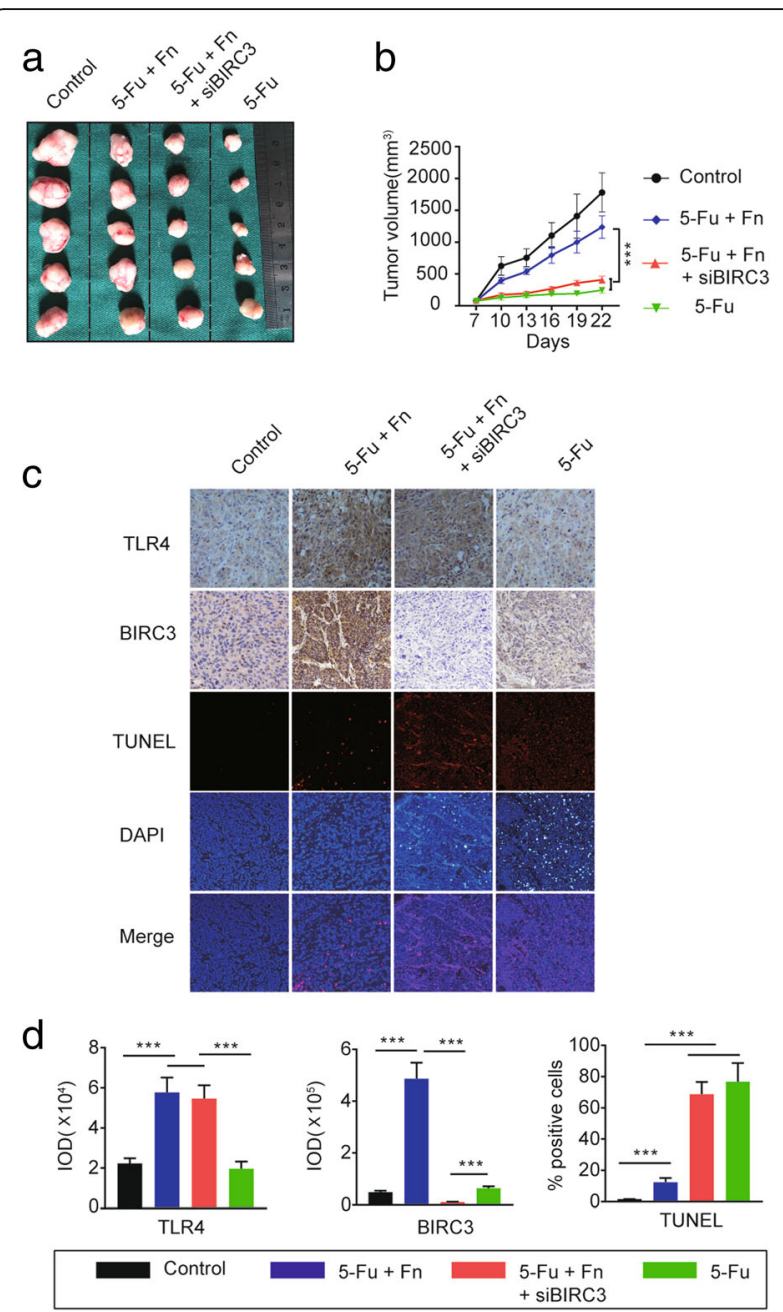

Fig. $4 \mathrm{Fn}$ induces chemoresistance of CRC cells in response to 5-Fu in vivo. a After the 2-week treatment, mice under different conditions were sacrificed and the tumors were collected and photographed. $\mathbf{b}$ Tumor sizes were measured every 3 days and tumor growth curves were established. Data are presented as tumor volume (mean \pm s.d.; ${ }^{* *} p<0.001$ by nonparametric Mann-Whitney test). c Immunohistochemical staining was carried out to detect the proteins expression levels of TLR4 and BIRC3, and a TUNEL assay was performed to detect tumor cell apoptosis in xenograft tumor tissues. $\mathbf{d}$ Integrated optical density (IOD) values were obtained using Image-Pro Plus software (Media Cybernetics, Rockville, MD, USA) and used to quantify the protein expression levels of TLR4 and BIRC3. Data are presented as IOD values. (mean \pm s.d.; ${ }^{* * *} p<0.001$ by nonparametric Mann-Whitney test). As for TUNEL assay, data are presented as the percentage of TUNEL- positive cells per high-power field of the various tumor sections. (mean \pm s.d.; ${ }^{* *} p<0.001$ by nonparametric Mann-Whitney test)

status (Table 1). Kaplan-Meier analysis showed that high amount of Fn (Fig. 5c, $p=0.028$ ) and high level of BIRC3 expression (Fig. 5d, $p=0.046$ ) correlated with poor RFS. However, the level of TLR4 expression was independent of RFS (Fig. 5e, $p=0.068$ ). Univariate Cox regression analyses for RFS were performed and the results showed that the risk of recurrence was associated with high $F n$ abundance $(\mathrm{HR}=2.02 ; 95 \% \mathrm{CI}=1.05$ to $3.88 ; p=0.03)$, positive vascular invasion $(\mathrm{HR}=2.72$; $95 \% \mathrm{CI}=1.49$ to $4.97 ; p=0.00)$ and positive neural invasion $(\mathrm{HR}=1.92 ; 95 \% \mathrm{CI}=1.02$ to $3.59 ; p=0.04$ ) (Fig. 5f). In the multivariate Cox regression analysis for RFS, the risk of recurrence was correlated with high amount of $F n(\mathrm{HR}=2.00 ; 95 \% \mathrm{CI}=1.01$ to $3.95 ; p=0.04)$ and positive vascular invasion $(\mathrm{HR}=2.73 ; 95 \% \mathrm{CI}=1.33$ to 5.60; $p=0.01$ ) (Fig. 5g). Taken together, these results demonstrate that high $F n$ abundance is an independent risk factor for recurrence in advanced CRC patients who received standard 5-Fu-based adjuvant chemotherapy after radical surgery, and further support that upregulation of BIRC3 expression induced by $F n$ might be responsible for chemoresistance in CRC.

\section{Discussion}

Fluorouracil-based adjuvant chemotherapy is a standard treatment for advanced CRC patients [41]. 5-Fu chemoresistance is a major challenge and the prognosis for CRC patients can be very poor due to recurrence of disease [42]. However, the potential mechanisms involved in the resistance to chemotherapeutic drugs including 5-Fu are not fully understood. It has been reported that drug absorption disorder, changes in drug targets, activation of DNA repair pathways, inhibition of apoptosis, and the tumor microenvironment are involved in drug resistance $[43,44]$. The mechanism of 5 -Fu resistance is associated with the activity of enzymes involved in the modulation of 5-Fu metabolism, such as thymidylate synthase, thymidine phosphorylase and dihydropyrimidine dehydrogenase [41]. Microsatellite instability status also correlates with chemosensitivity to 5-Fu [45]. Interestingly, increasing evidence suggests that gut microbiota may affect the efficacy of antitumor drugs [10, 46]. In this study, we initially found that BIRC3 was the most upregulated gene induced by $F n$ infection in CRC cell lines. Moreover, BIRC3 is a member of the IAP family that inhibits apoptosis by directly inhibiting the caspase cascade $[24,25]$, which has potential to aid in the treatment of CRC [19]. As inhibition of apoptosis is an important mechanism of drug resistance [47], we speculated that the upregulation of BIRC3 induced by $F n$ may be associated with chemoresistance. It is well acknowledged that 5 - Fubased chemotherapy is the principal postoperative treatment for advanced CRC [48]. Therefore, we hypothesized that $F n$ infection and upregulation of BIRC3 may be involved in resistance to 5 -Fu. To test this hypothesis, we first demonstrated that $F n$ infection reduced the chemosensitivity of CRC cells to 5-Fu in vitro and in vivo. Next, we confirmed that this biological effect depends on the upregulation of BIRC3. Furthermore, we dissected the mechanisms involved in Fn-induced BIRC3 expression, 


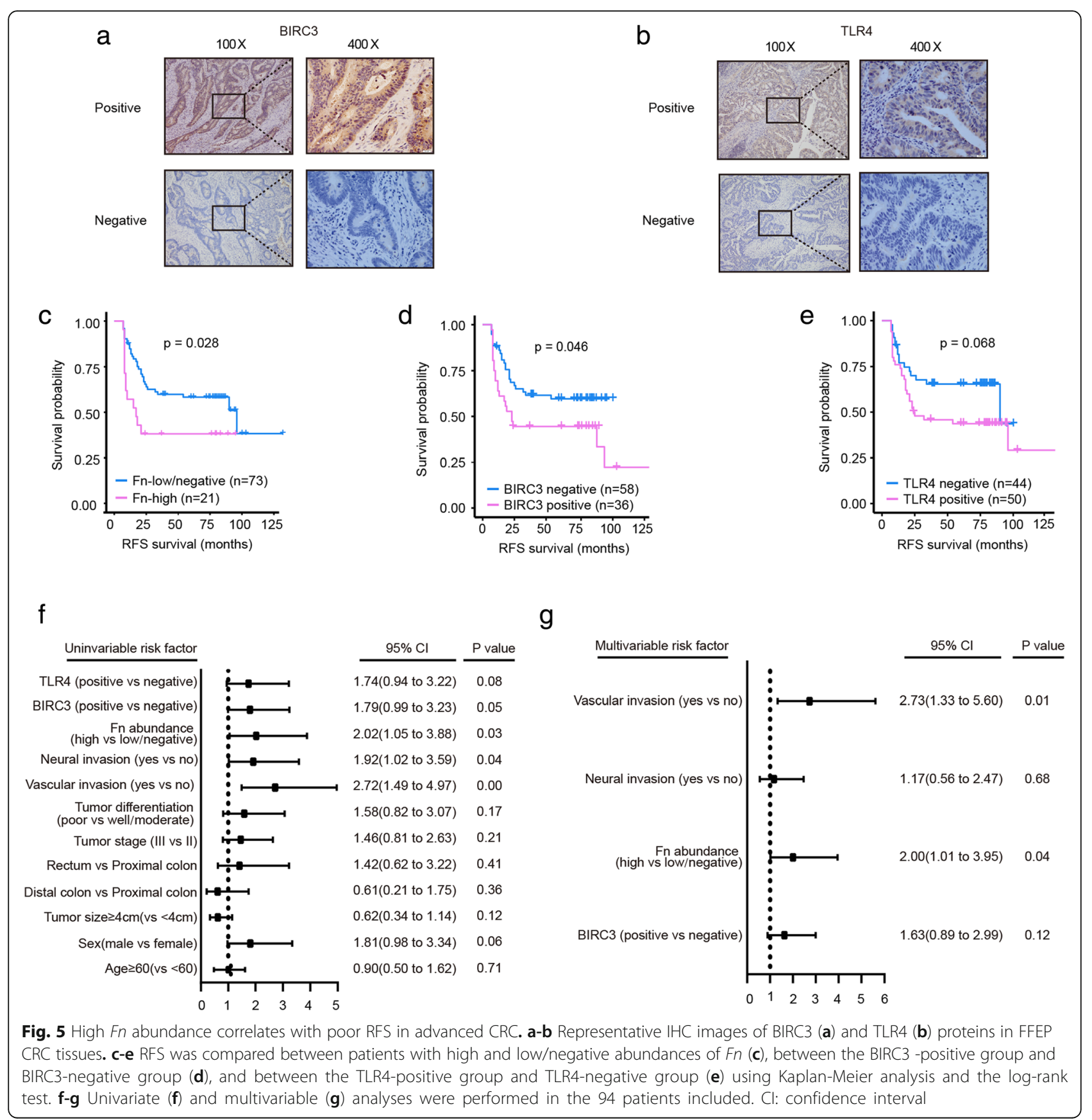

which showed that the TLR4/NF-kB pathway regulates BIRC3 expression in CRC cells that are cocultured with $F n$. Finally, to correspond to the aforementioned findings, we investigated the clinical significance of $F n$ infection and its downstream target genes. The results showed that a high amount of $F n$ was an independent risk factor for recurrence in advanced CRC patients.

Many studies have reported that overexpression of BIRC3 is associated with chemoresistance in the treatment of malignancies. Karasawa et al. found that BIRC3positive patients had a shorter disease-free survival after
5-Fu-based chemotherapy [26] and suggested that BIRC3 can serve as a therapeutic target in CRC and other malignancies [49]. Krajewska et al. found that elevated expression of BIRC3 significantly correlated with shorter overall survival in early-stage CRC [50]. In addition, overexpression of BIRC3 significantly correlated with resistance to several chemotherapeutic drugs, including $5-\mathrm{Fu}$, in pancreatic cancer cells [34]. Upregulation of BIRC3 induced by $\mathrm{IL}-1 \beta$ results in chemoresistance to doxorubicin in breast cancer cells [51]. Consistent with these findings, our results showed that a high level of BIRC3 induced by 


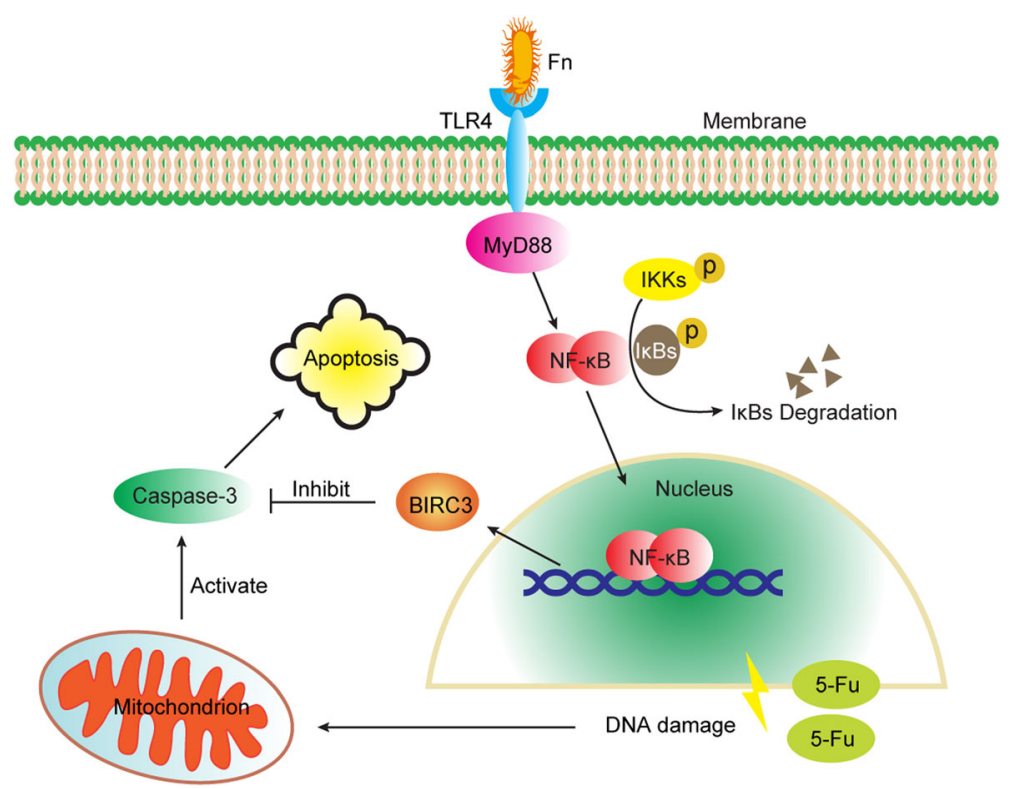

Fig. 6 Schematic diagram of the mechanism by which Fn protects CRC cells from 5-Fu-mediated apoptosis via upregulation of BIRC3 expression

$F n$ infection contributed to chemoresistance to $5-\mathrm{Fu}$ in vitro, and correlated with poor RFS in CRC patients. A recent study reported that $F n$ can mediate chemoresistance by autophagy pathway activation in CRC [52]. In line with this, our study observed similar phenomena elucidated with different mechanism. Another study found that $F n$ can migrate with CRC cells to metastatic sites and that CRC patients may benefit from eradication of $F n$ with antibiotic treatment [53]. Therefore, we may infer that $F n$ as a part of metastatic tumor plays a role in the chemoresistance to 5 - Fu treatment and contributes to disease recurrence. However, there are some limitations in this research. First, this study just focuses on $F n$ enriched in tumor tissues and the indirect effect of $F n$ on 5 -Fu. It is still unknown that whether $F n$ inhabiting in intestinal lumen of CRC patients can directly affect the efficacy of 5-Fu. Second, subcutaneous xenograft cannot simulate intestinal microenvironment very well. So, further study is needed to investigate the direct interactions between $\mathrm{Fn}$ and chemotherapeutic drugs.

\section{Conclusions}

In conclusion, our study initially found that $F n$ infection reduces the chemosensitivity of $\mathrm{CRC}$ cells to 5 -Fu through the upregulation of BIRC3, and that the TLR4/ NF- $\mathrm{kB}$ pathway regulates BIRC3 expression in CRC cells cocultured with $F n$ (Fig. 6). Targeting $F n$ infection and BIRC3 may be promising in the treatment of advanced CRC patients who received 5-Fu-based chemotherapy after radical surgery, and may optimize the current treatment strategy of CRC.

\section{Additional files}

Additional file 1: Table S1. Primer sequences of $\mathrm{qPCR}$ and $C h \mid \mathrm{P}-\mathrm{qPCR}$ and siRNA sequences used in the study. (XLSX $10 \mathrm{~kb}$ )

Additional file 2: Table S2. Information of antibody used in the study. (XLSX $9 \mathrm{~kb}$ )

Additional file 3: Figure S1. The peak map of DNA sequencing about the constructions of wild-type and mutant BIRC3 promoters. (PDF $712 \mathrm{~kb}$ )

Additional file 4: Figure S2. Distribution of $F n$ abundance in CRC patients $(n=94)$. The relative abundance of $F n$ in FFPE CRC tissues was determined by $2^{-\Delta c t}$. The patients were ranked according to abundance of Fn. A cut-off value of 0.1 was set to distinguish the high $F n$ abundance group ( $n=21)$ from the low/negative Fn abundance group $(n=73)$. (TIF $508 \mathrm{~kb})$

\section{Abbreviations}

5-Fu: 5-fluorouracil; ATCC: American Type Culture Collection; CCK8: Cell Counting Kit-8; ChIP: Chromatin immunoprecipitation assay; CRC: Colorectal cancer; FFPE: Formalin-fixed paraffin-embedded; Fn: Fusobacterium nucleatum; gDNA: Genomic DNA; IAP: Inhibitor of apoptosis protein; $I_{50}$ : 50\% inhibitory concentration; IHC: Immunohistochemistry; MOI: Multiplicity of infection; MT: Mutant type; PGT: Gene prostaglandin transporter; qPCR: Quantitative real-time polymerase chain reaction; RFS: Recurrence-free survival; siRNA: Small interfering RNA; TF-NC: Transcription factor-negative control; TF-P65: Transcription factor-P65; TSS: Transcription start site; TUNEL: Terminal deoxynucleotidyl transferase-mediated dUTP nick end labeling; WT: Wild-type

\section{Acknowledgements}

Not applicable.

\section{Funding}

This work was supported by grants from the National Natural Science Foundation of China (No.81001069). Fudan Outstanding Young Talent Training Plan (No.YJYQ201601), and Shanghai Pujiang Program (No.17PJD007).

Availability of data and materials

All data are included in the manuscript and the additional files. 


\section{Authors' contributions}

SC and YM conceived the experiments. SZ, YY, WW and BG conducted the experiments. SZ and GC analyzed and interpreted the data. SZ wrote the manuscript and prepared the figures. All authors read and approved the final manuscript.

\section{Ethics approval and consent to participate}

This study was approved by the Ethics Committee of Fudan Cancer Hospital and in accordance with the ethical standards formulated in the Declaration of Helsinki. Informed consent was obtained from all patients included in the study. All animal studies were approved by the Committee on Animals Handling of Fudan University Shanghai Cancer Center.

\section{Consent for publication}

All authors approved of the manuscript and consented to its publication.

\section{Competing interests}

The authors declare that have no competing interests.

\section{Publisher's Note}

Springer Nature remains neutral with regard to jurisdictional claims in published maps and institutional affiliations.

\section{Author details}

'Department of Colorectal Surgery, Fudan University Shanghai Cancer Center, 270 Dong'an Road, Shanghai 200032, China. ${ }^{2}$ Department of Oncology, Shanghai Medical College, Fudan University, Shanghai 200032, China. ${ }^{3}$ Department of Clinical Laboratory, Yangpu Hospital, Tongji University School of Medicine, Shanghai, China. ${ }^{4}$ Center for Translational Medicine, Yangpu Hospital, Tongji University School of Medicine, Shanghai, China. ${ }^{5}$ Department of Surgery, Shanghai Jiao Tong University Affiliated Sixth People's Hospital, Shanghai, China.

\section{Received: 26 October 2018 Accepted: 29 November 2018} Published online: 10 January 2019

\section{References}

1. Torre LA, Bray F, Siegel RL, Ferlay J, Lortet-Tieulent J, Jemal A. Global cancer statistics, 2012. CA Cancer J Clin. 2015;65(2):87-108.

2. Chen W, Zheng R, Baade PD, Zhang S, Zeng H, Bray F, Jemal A, Yu XQ, He J. Cancer statistics in China, 2015. CA Cancer J Clin. 2016;66(2):115-32.

3. Garcia-Castillo V, Sanhueza E, McNerney E, Onate SA, Garcia A. Microbiota dysbiosis: a new piece in the understanding of the carcinogenesis puzzle. J Med Microbiol. 2016;65(12):1347-62.

4. Wu N, Yang X, Zhang R, Li J, Xiao X, Hu Y, Chen Y, Yang F, Lu N, Wang Z, et al. Dysbiosis signature of fecal microbiota in colorectal cancer patients. Microb Ecol. 2013;66(2):462-70.

5. Louis P, Hold GL, Flint HJ. The gut microbiota, bacterial metabolites and colorectal cancer. Nat Rev Microbiol. 2014;12(10):661-72.

6. Sivan A, Corrales L, Hubert N, Williams JB, Aquino-Michaels K, Earley ZM, Benyamin FW, Lei YM, Jabri B, Alegre ML, et al. Commensal Bifidobacterium promotes antitumor immunity and facilitates anti-PD-L1 efficacy. Science. 2015;350(6264):1084-9.

7. Vetizou M, Pitt JM, Daillere R, Lepage P, Waldschmitt N, Flament C, Rusakiewicz S, Routy B, Roberti MP, Duong CP, et al. Anticancer immunotherapy by CTLA-4 blockade relies on the gut microbiota. Science. 2015;350(6264):1079-84.

8. Zitvogel L, Ayyoub M, Routy B, Kroemer G. Microbiome and Anticancer Immunosurveillance. Cell. 2016;165(2):276-87.

9. Routy B, Gopalakrishnan V, Daillere R, Zitvogel L, Wargo JA, Kroemer G. The gut microbiota influences anticancer immunosurveillance and general health. Nat Rev Clin Oncol. 2018;15(6):382-96.

10. Coleman Ol, Nunes T. Role of the microbiota in colorectal Cancer: updates on microbial associations and therapeutic implications. Biores Open Access. 2016:5(1):279-88.

11. Scott TA, Quintaneiro LM, Norvaisas P, Lui PP, Wilson MP, Leung KY, HerreraDominguez L, Sudiwala S, Pessia A, Clayton PT, et al. Host-microbe cometabolism dictates Cancer drug efficacy in C. elegans. Cell. 2017:169(3): 442-56 e18.
12. Garcia-Gonzalez AP, Ritter AD, Shrestha S, Andersen EC, Yilmaz LS, Walhout AJM. Bacterial metabolism affects the $C$. elegans response to Cancer chemotherapeutics. Cell. 2017;169(3):431-41 e8.

13. Castellarin M, Warren RL, Freeman JD, Dreolini L, Krzywinski M, Strauss J, Barnes R, Watson P, Allen-Vercoe E, Moore RA, et al. Fusobacterium nucleatum infection is prevalent in human colorectal carcinoma. Genome Res. 2012;22(2):299-306.

14. Mima K, Nishihara R, Qian ZR, Cao Y, Sukawa Y, Nowak JA, Yang J, Dou R, Masugi $Y$, Song $M$, et al. Fusobacterium nucleatum in colorectal carcinoma tissue and patient prognosis. Gut. 2016;65(12):1973-80.

15. Gur C, Ibrahim Y, Isaacson B, Yamin R, Abed J, Gamliel M, Enk J, Bar-On Y, Stanietsky-Kaynan N, Coppenhagen-Glazer S, et al. Binding of the Fap2 protein of fusobacterium nucleatum to human inhibitory receptor TIGIT protects tumors from immune cell attack. Immunity. 2015;42(2):344-55.

16. Rubinstein MR, Wang X, Liu W, Hao Y, Cai G, Han YW. Fusobacterium nucleatum promotes colorectal carcinogenesis by modulating E-cadherin/ beta-catenin signaling via its FadA adhesin. Cell Host Microbe. 2013;14(2): 195-206.

17. Yang $Y$, Weng W, Peng J, Hong L, Yang L, Toiyama Y, Gao R, Liu M, Yin M, Pan C, et al. Fusobacterium nucleatum increases proliferation of colorectal Cancer cells and tumor development in mice by activating toll-like receptor 4 signaling to nuclear factor-kappaB, and up-regulating expression of MicroRNA-21. Gastroenterology. 2017;152(4):851-66 e24.

18. Zhang S, Cai S, Ma Y. Association between fusobacterium nucleatum and colorectal cancer: Progress and future directions. J Cancer. 2018;9(9):1652-9.

19. Miura K, Fujibuchi W, Ishida K, Naitoh T, Ogawa H, Ando T, Yazaki N, Watanabe K, Haneda S, Shibata C, et al. Inhibitor of apoptosis protein family as diagnostic markers and therapeutic targets of colorectal cancer. Surg Today. 2011;41(2):175-82.

20. Deveraux QL, Reed JC. IAP family proteins--suppressors of apoptosis. Genes Dev. 1999;13(3):239-52.

21. Altieri DC. Survivin and IAP proteins in cell-death mechanisms. Biochem J. 2010;430(2):199-205.

22. Wang CY, Cusack JC Jr, Liu R, Baldwin AS Jr. Control of inducible chemoresistance: enhanced anti-tumor therapy through increased apoptosis by inhibition of NF-kappaB. Nat Med. 1999:5(4):412-7.

23. LaCasse EC, Mahoney DJ, Cheung HH, Plenchette S, Baird S, Korneluk RG. IAP-targeted therapies for cancer. Oncogene. 2008;27(48):6252-75.

24. Wang CY, Mayo MW, Korneluk RG, Goeddel DV, Baldwin AS Jr. NF-kappaB antiapoptosis: induction of TRAF1 and TRAF2 and C-IAP1 and C-IAP2 to suppress caspase-8 activation. Science. 1998;281(5383):1680-3.

25. Park SM, Yoon JB, Lee TH. Receptor interacting protein is ubiquitinated by cellular inhibitor of apoptosis proteins (C-IAP1 and c-IAP2) in vitro. FEBS Lett. 2004:566(1-3):151-6.

26. Karasawa H, Miura K, Fujibuchi W, Ishida K, Kaneko N, Kinouchi M, Okabe M, Ando T, Murata Y, Sasaki H, et al. Down-regulation of CIAP2 enhances 5-FU sensitivity through the apoptotic pathway in human colon cancer cells. Cancer Sci. 2009;100(5):903-13.

27. Mendes RT, Nguyen D, Stephens D, Pamuk F, Fernandes D, Van Dyke TE, Kantarci A. Endothelial cell response to fusobacterium nucleatum. Infect Immun. 2016;84(7):2141-8.

28. Livak KJ, Schmittgen TD. Analysis of relative gene expression data using real-time quantitative PCR and the 2(-Delta Delta C(T)) method. Methods. 2001;25(4):402-8.

29. Huang L, Zhai E, Cai S, Lin Y, Liao J, Jin H, Peng $S, X u$ L, Chen M, Zeng Z. Stress-inducible Protein-1 promotes metastasis of gastric cancer via Wnt/ beta-catenin signaling pathway. J Exp Clin Cancer Res. 2018;37(1):6.

30. Wang L, Shi S, Guo Z, Zhang X, Han S, Yang A, Wen W, Zhu Q. Overexpression of YAP and TAZ is an independent predictor of prognosis in colorectal cancer and related to the proliferation and metastasis of colon cancer cells. PLoS One. 2013;8(6):e65539.

31. Xu Z, Lin S, Wu W, Tan H, Wang Z, Cheng C, Lu L, Zhang X. Ghrelin prevents doxorubicin-induced cardiotoxicity through TNF-alpha/NF-kappaB pathways and mitochondrial protective mechanisms. Toxicology. 2008;247(2-3):133-8.

32. Wang F, Zhang P, Ma Y, Yang J, Moyer MP, Shi C, Peng J, Qin H. NIRF is frequently upregulated in colorectal cancer and its oncogenicity can be suppressed by let-7a microRNA. Cancer Lett. 2012;314(2):223-31.

33. Duckett CS, Nava VE, Gedrich RW, Clem RJ, Van Dongen JL, Gilfillan MC, Shiels $\mathrm{H}$, Hardwick JM, Thompson CB. A conserved family of cellular genes related to the baculovirus iap gene and encoding apoptosis inhibitors. EMBO J. 1996;15(11):2685-94. 
34. Lopes RB, Gangeswaran R, McNeish IA, Wang Y, Lemoine NR. Expression of the IAP protein family is dysregulated in pancreatic cancer cells and is important for resistance to chemotherapy. Int J Cancer. 2007;120(11): 2344-52.

35. Fulda S. Promises and challenges of Smac mimetics as Cancer therapeutics. Clin Cancer Res. 2015;21(22):5030-6.

36. Duckett CS. IAP proteins: sticking it to Smac. Biochem J. 2005;385(1):e1-2.

37. Abreu MT, Peek RM Jr. Gastrointestinal malignancy and the microbiome. Gastroenterology. 2014;146(6):1534-46 e3.

38. Wang $Q$, Wang $X$, Evers BM. Induction of CIAP-2 in human colon cancer cells through PKC delta/NF-kappa B. J Biol Chem. 2003;278(51):51091-9.

39. Chu ZL, McKinsey TA, Liu L, Gentry JJ, Malim MH, Ballard DW. Suppression of tumor necrosis factor-induced cell death by inhibitor of apoptosis c-IAP2 is under NF-kappaB control. Proc Natl Acad Sci U S A. 1997;94(19):10057-62.

40. Tahara T, Yamamoto E, Suzuki H, Maruyama R, Chung W, Garriga J, Jelinek J, Yamano HO, Sugai T, An B, et al. Fusobacterium in colonic flora and molecular features of colorectal carcinoma. Cancer Res. 2014;74(5):1311-8.

41. Longley DB, Harkin DP, Johnston PG. 5-fluorouracil: mechanisms of action and clinical strategies. Nat Rev Cancer. 2003;3(5):330-8.

42. Brenner H, Kloor M, Pox CP. Colorectal cancer. Lancet. 2014;383(9927):1490-502.

43. Sheikh R, Walsh N, Clynes M, O'Connor R, McDermott R. Challenges of drug resistance in the management of pancreatic cancer. Expert Rev Anticancer Ther. 2010;10(10):1647-61.

44. Niero EL, Rocha-Sales B, Lauand C, Cortez BA, de Souza MM, RezendeTeixeira P, Urabayashi MS, Martens AA, Neves JH, Machado-Santelli GM. The multiple facets of drug resistance: one history, different approaches. J Exp Clin Cancer Res. 2014;33:37

45. Sargent DJ, Marsoni S, Monges G, Thibodeau SN, Labianca R, Hamilton SR, French AJ, Kabat B, Foster NR, Torri V, et al. Defective mismatch repair as a predictive marker for lack of efficacy of fluorouracil-based adjuvant therapy in colon cancer. J Clin Oncol. 2010;28(20):3219-26.

46. lida N, Dzutsev A, Stewart CA, Smith L, Bouladoux N, Weingarten RA, Molina DA, Salcedo R, Back T, Cramer S, et al. Commensal bacteria control cancer response to therapy by modulating the tumor microenvironment. Science. 2013;342(6161):967-70

47. Baguley BC. Multiple drug resistance mechanisms in cancer. Mol Biotechnol. 2010:46(3):308-16

48. Midgley R, Kerr D. Colorectal cancer. Lancet. 1999;353(9150):391-9.

49. Miura K, Karasawa H, Sasaki I. cIAP2 as a therapeutic target in colorectal cancer and other malignancies. Expert Opin Ther Targets. 2009;13(11):1333-45.

50. Krajewska M, Kim H, Kim C, Kang H, Welsh K, Matsuzawa S, Tsukamoto M, Thomas RG, Assa-Munt N, Piao Z, et al. Analysis of apoptosis protein expression in early-stage colorectal cancer suggests opportunities for new prognostic biomarkers. Clin Cancer Res. 2005;11(15):5451-61.

51. Mendoza-Rodriguez M, Arevalo Romero H, Fuentes-Panana EM, AyalaSumuano JT, Meza I. IL-1 beta induces up-regulation of BIRC3, a gene involved in chemoresistance to doxorubicin in breast cancer cells. Cancer Lett. 2017:390:39-44

52. Yu T, Guo F, Yu Y, Sun T, Ma D, Han J, Qian Y, Kryczek I, Sun D, Nagarsheth $\mathrm{N}$, et al. Fusobacterium nucleatum promotes Chemoresistance to colorectal Cancer by modulating autophagy. Cell. 2017;170(3):548-63 e16.

53. Bullman S, Pedamallu CS, Sicinska E, Clancy TE, Zhang X, Cai D, Neuberg D, Huang K, Guevara F, Nelson T, et al. Analysis of fusobacterium persistence and antibiotic response in colorectal cancer. Science. 2017;358(6369):1443-8.

Ready to submit your research? Choose BMC and benefit from:

- fast, convenient online submission

- thorough peer review by experienced researchers in your field

- rapid publication on acceptance

- support for research data, including large and complex data types

- gold Open Access which fosters wider collaboration and increased citations

- maximum visibility for your research: over $100 \mathrm{M}$ website views per year

At BMC, research is always in progress.

Learn more biomedcentral.com/submissions 